\title{
POSITIVE DEFINITENESS OF PIECEWISE-LINEAR FUNCTION 2
}

\author{
AnATOLiY MANOV
}

Abstract. Let $\alpha, \beta \in(0,1), 0<\alpha \leqslant \beta<1, s \in \mathbb{R}$ and let $w_{\alpha, \beta, s}$ be an even function with the properties: $w_{\alpha, \beta, s}(x)=0$ for $x>1, w_{\alpha, \beta, s}(0)=1, w_{\alpha, \beta, s}(1)=0, w_{\alpha, \beta, s}(x)=s$ for $x \in[\alpha, \beta]$ $([\alpha, \alpha]:=\{\alpha\}), w_{\alpha, \beta, s}$ is linear over the intervals $[0, \alpha]$ and $[\beta, 1]$. In this paper we prove that $w_{\alpha, \beta, s}$ is positive definite on $\mathbb{R} \Longleftrightarrow m(\alpha, \beta) \leqslant s \leqslant M(\alpha, \beta)$, where $m(\alpha, \beta) \leqslant 0, M(\alpha, \beta) \geqslant 0$. If either $(1+\beta) / \alpha,(1-\beta) / \alpha \in \mathbb{N}$ or $1 / \alpha \notin \mathbb{N}, \beta / \alpha \in \mathbb{N}$, then $M(\alpha, \beta)>0$, otherwise $M(\alpha, \beta)=0$. If either $(1+\beta) / \alpha,(1-\beta) / \alpha \in \mathbb{N}$ or $1 / \alpha \in \mathbb{N}, \beta / \alpha \notin \mathbb{N}$, then $m(\alpha, \beta)<0$, otherwise $m(\alpha, \beta)=0$. Moreover, we find explicit values of $M(\alpha, \beta), m(\alpha, \beta)$ for some $\alpha$ and $\beta$.

Mathematics subject classification (2010): 42A82.

Keywords and phrases: Positive-definite functions, piecewise-linear functions, Bochner theorem, completely monotone functions.

\section{REFERENCES}

[1] N. I. AKHIEZeR, Lectures on Integral Transforms, American Mathematical Society, Providence, Rhode Island, 1988.

[2] R. A. Horn, C. R. Johnson, Matrix analysis, Cambridge University Press, Cambridge, London, New York, 1985.

[3] P. Jorgensen, S. Pedersen, F. Tian, Extensions of Positive Definite Functions, Springer, Heidelberg, 2016.

[4] E. LuKacs, Characteristic Functions, Griffin, London, 1970.

[5] A. Manov, V. Zastavnyi, Positive definiteness of piecewise-linear function, Expo. Math., 35, 3, 2017, 357-361.

[6] Z. SASVÁRI, Multivariate Characteristic and Correlation Functions, De Gruyter, Berlin, Boston, 2013.

[7] R. M. Trigub, E. S. Belinsky, Fourier Analysis and Approximation of Functions, KluwerSpringer, Boston, Dordrecht, London, 2004.

[8] V. P. Zastavnyi, A. D. Manov, Positive definiteness of complex piecewise-linear function and some of its applications, Math. Notes., 103, 4, 2018.

[9] V. P. ZastaVnYI, Positive definite functions and sharp inequalities for periodic functions, Ural Mathematical Journal, 3, 2, 2017, 82-99.

[10] V. P. Zastavnyi, On positive definiteness of some functions, Journal of Multivariate Analysis, 73, 1, 2000, 55-81. 